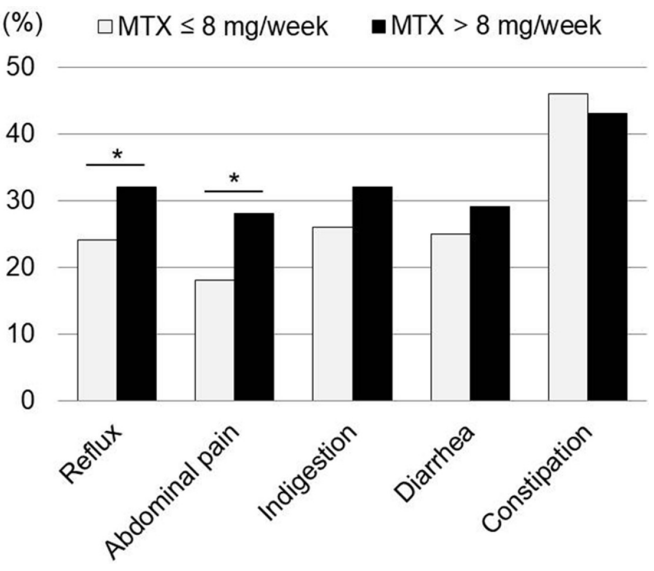

Abstract THU0176 - Figure 1. Percentage of patients with GSRS score $\geq 2$.

Conclusions: MTX more than $8 \mathrm{mg} /$ week is associated with upper Gl symptoms as much as NSAIDs in Japanese patients with RA.

Disclosure of Interest: None declared

DOI: 10.1136/annrheumdis-2018-eular.1715

\section{THU0177 CAN SIMPLE EDUCATIONAL FLASHCARDS CHANGE ATTITUDES TO VACCINATION IN AN UNDER- VACCINATED RHEUMATOID ARTHRITIS COHORT?}

S. Raghunath ${ }^{1,2}$, T. Kovitwanichkanont ${ }^{1,3}$, D. Wang $^{2}$, N. Walpola ${ }^{2}$, L. Kyi ${ }^{1}$, S. Pignataro ${ }^{1}$, S. Morton ${ }^{1}$, E. Thompson ${ }^{2}$, M. Leech ${ }^{1,2} .{ }^{1}$ Monash Health; ${ }^{2}$ Monash University, Melbourne; ${ }^{3}$ University of Sydney, Sydney, Australia

Background: There is increased infection risk in RA which relates to immune system dysregulation, comorbidities and immunosuppression. ${ }^{1,2}$ Therefore, there is a need to improve currently suboptimal vaccination rates reported globally. ${ }^{3}$ Objectives: To re-examine vaccination rates and to determine if educational flashcards with messages targeted to patient perceptions can change attitudes towards vaccination in a tertiary hospital RA clinic cohort.

Methods: Vaccination status and attitudes were examined in 126 consecutive rheumatoid arthritis clinic patients (data collection ongoing). Patients were then shown two simple educational flashcards and any shift in attitude to vaccination was recorded. Insights gleaned from a 2016 study on attitudes to vaccination in the same cohort were used to design the flashcards based on unvaccinated patients' main concerns. ${ }^{4}$

Results: The RA cohort was representative of a typical RA population. $67 \%$ of patients were female with a mean age of 57 years (range 18 to 88 years). $40 \%$ of patients were on biologic medication and $34 \%$ were on prednisolone. $13 \%$ of patients were previously hospitalised for influenza or pneumonia and $15 \%$ had a close contact hospitalised for these infections. $37 \%$ of patients had not received the influenza vaccine. In patients over 65 years old, $51 \%$ had not received the pneumococcal vaccine within the last 5 years, and of those, $80 \%$ had never been vaccinated against pneumococcal. Reasons reported for not being vaccinated included "I forget", "I worry about the side effects of vaccine", "I don't think I need the vaccine as I don't get the flu", and "I had the vaccine and it made me sick". $33 \%$ of patients were not aware of the hospitals free vaccination service, and of those that were aware of this service $13 \%$ cited this as their main reason for getting the vaccine.

After reading the education flashcards $49 \%$ of currently unvaccinated patients reported "I feel more informed and am more likely to get the vaccine next year". Rates of vaccination, infection and attitudes both pre and post flashcards did not differ between patients on biologics and not on biologics (all p-values $>0.05$ ).

\begin{tabular}{lc}
\hline \begin{tabular}{l} 
Reasons reported for not vaccinating (in order of frequency) \\
\hline 1
\end{tabular} & I forgot \\
\hline 2 & I am worried about the side effects of the vaccine. \\
3 & I don't think I need the vaccine as I don't get the flu. \\
4 & I don't think the vaccine will work., \\
5 & I have received it before so I don't need it or want it this \\
6 & year. \\
7 & I am afraid of needles \\
\hline
\end{tabular}

Vaccine Education Flashcards

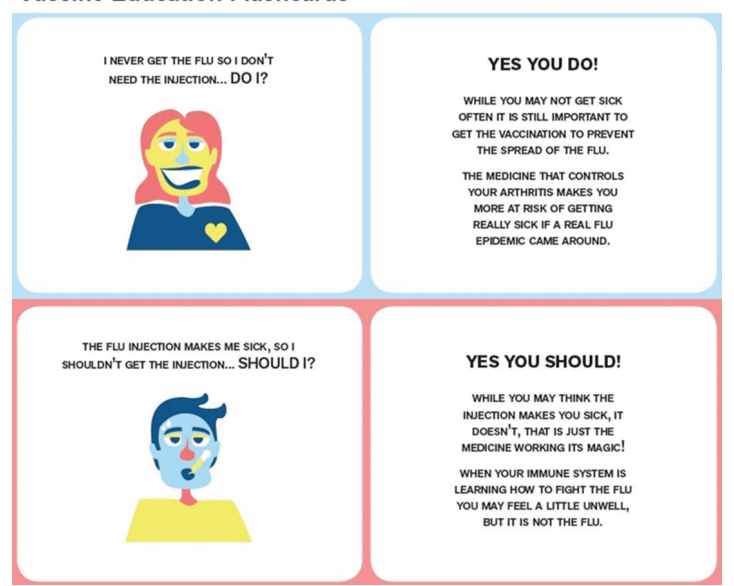

Conclusions: This at-risk RA cohort continues to be under-vaccinated in studies over the past two years. Simple flashcards showed potential to change attitudes in unvaccinated patients who were previously comfortable to express resistance. Insights from this study could be used to refine and reiterate this educational inter vention for implementation in a larger cohort to measure impact on vaccination rates in subsequent years.

\section{REFERENCES :}

[1] Doran M, et al. Arthritis \& Rheumatology 2002;46(9):2287-2293.

[2] Doran M, et al. Arthritis \& Rheumatology 2002;46(9):2294-2300.

[3] Hmamouchi I, et al. Vaccine 2015;33(12):1446-1452.

[4] Jh L, et al. Global Vaccines 2016;2.

Disclosure of Interest: None declared DOI: 10.1136/annrheumdis-2018-eular.4030

\section{THU0178 CLINICAL SIGNIFICANCE OF PERIODONTITIS IN RHEUMATOID ARTHRITIS PATIENTS: ASSOCIATION WITH DISEASE ACTIVITY, FUNCTIONAL STATUS AND RADIOLOGRAPHIC SCORE}

T.A. Gheita ${ }^{1}$, H.M. Fathi ${ }^{2}$, N. Khairy ${ }^{3}$, W.A. El-Battawy ${ }^{4} .{ }^{1}$ Rheumatology and Clinical Immunology, Faculty of Medicine, Kasr Al-Ainy School of Medicine, Cairo University, Cairo; ${ }^{2}$ Rheumatology, Faculty of Medicine, Fayoum University, Fayoum; ${ }^{3}$ Rheumatology, Faculty of Medicine, Cairo University; ${ }^{4}$ Oral Medicine and Periodontology, Faculty of Oral and Dental Medicine, Cairo University, Cairo, Egypt

\section{Background:}

Objectives: To evaluate frequency of periodontitis (PD) in rheumatoid arthritis (RA) patients and relate it with clinical characteristics, disease activity, functional status, anti-cyclic citrullinated peptide (anti-CCP) and radiographic scores.

Methods: The study included 60 RA patients and 30 controls. Clinical Disease activity index (CDAI), Modified Health Assessment Questionnaire (MHAQ), visual analogue scale of pain and Scott's modification to Larsen scoring method were assessed. Rheumatoid factor (RF) positivity and antiCCP titer were measured. Periodontal examination was performed and relevant indeces calculated

Results: The mean age of the patients was $49.1 \pm 13$ years and they were 52 females and 8 males. PD was present in $71.7 \%$ of RA patients versus $46.7 \%$ in control $(p=0.02)$. PD was predominantly generalised $(p=0.004)$ and moderatesevere degree $(p=0.01)$. Age $(p=0.007)$, disease duration $(p<0.0001)$, morning stiffness $(p=0.01)$, CDAI $(p<0.0001), \operatorname{MHAQ}(p=0.02)$, CRP $(p=0.02)$, anti-CCP titer $(p=0.01)$ and methotrexate treatment $(p=0.005)$ were significantly higher in RA-PD versus RA. However, gender, smoking, oral hygiene, erythrocyte sedimentation rate, $\mathrm{RF}$, anti-CCP positivity and radiographic scoring were insignificantly different. PD positivity was $96.3 \%$, predominant generalised in $92.6 \%$, moderate $(40.7 \%)$ and severe degree $(37 \%)$ in early RA versus $(51.5 \%, 24.2 \%, 24.2 \%, 12.1 \%$ respectively) in late RA patients. All PD indices were higher in early patients $(p \leq 0.05)$ while teeth loss $(p=0.03)$ was higher in late cases. CDAI, VAS and ACPA titer all signficantly correlated with PD indices $(p<0.05)$ Conclusions: Periodontitis is frequent in RA patients' especially in early cases and is remarkably associated to disease activity and reduced functional status.

Disclosure of Interest: None declared

DOI: 10.1136/annrheumdis-2018-eular.5201 